\title{
A computer aided tolerancing tool II: Tolerance analysis
}

\author{
O.W. Salomons ${ }^{*}$, F.J. Haalboom, H.J. Jonge Poerink, F. van Slooten, \\ F.J.A.M. van Houten, H.J.J. Kals
}

University of Twente, Department of Mechanical Engineering, Laboratory of Production and Design Engineering, P.O. Box 217,7500 AE Enschede, The Netherlands

Received 1 February 1995; revised 4 July 1996; accepted 4 July 1996

\begin{abstract}
A computer aided tolerance analysis tool is presented that assists the designer in evaluating worst case quality of assembly after tolerances have been specified. In tolerance analysis calculations, sets of equations are generated. The number of equations can be restricted by using a minimum number of points in which quality of assembly is calculated. The number of points needed depends on the type of surface association. The number of parameters in the set of equations can be reduced by considering the most critical direction for the assembly condition. The latter direction, called virtual plan fragment direction, is determined using a virtual plan fragment table, based on an analogy to the plan fragment lable used in degrees of freedom (DOF) analysis. This reduced set of equations is then solved and optimized in order to find the maximum/minimum values for the assembly condition using simulated annealing. This method for tolerance analysis has been implemented in a feature based (re-)design support system called FROOM, as part of the functional tolerancing module.
\end{abstract}

Keywords: Tolerance analysis; TTRS; Simulated annealing

\section{Introduction}

This paper is the second in a series of two on a computer aided tolerancing tool as part of a larger academic re-design support system prototype called FROOM. In this paper tolerance analysis is stressed whereas in the first paper - part J. - tolerance specification was the main topic. An introduction to tolerance analysis is provided in Section 1.1. The FROOM system, whose tolerancing module is the focus of the remainder of this paper is introduced in Section 1.2. Section 1.3 provides an overview of the remainder of this paper.

\subsection{Tolerance analysis}

After tolerance specification (see the first paper; part I), and checking the coherence and completeness of the specified tolerances, tolerance analysis can be performed. Tolerance analysis is a method to verify the proper

\footnotetext{
* Corresponding author. Email: o.w.salomons@wb.utwente.nl 
functioning of the assembly after tolerances have been specified. Most often, tolerance analysis is performed by verifying two aspects: feasibility and quality of assembly. In feasibility of assembly the fit is verified. In quality of assembly clearances are verified. Feasibility of assembly can be considered by checking face associations of faces of two different components which influence fit, two by two, without considering other faces in a part or in the remainder of the mechanism. In quality of assembly, clearances and orientations between two surfaces of two distinct parts that take part in a tolerance chain or loop are calculated. In order to perform such calculations all the tolerances that are part of the tolerance chain/loop have to be considered. The designer has manually specified upper and lower values for clearances and orientations between different parts in an assembly in so-called assembly conditions; sec the tolerance specification paper (part I). The clcarances and oricntations specified in these assembly conditions are of importance for the functioning of the assembly. For example, in a gearpump, the clearance between the two gearwheels determines the functioning of the assembly. It is obvious, that quality of assembly is difficult to calculate, especially when $3 \mathrm{D}$ cases have to be considered. This is because the positions of the surfaces of the two distinct parts should be calculated with respect to the coordinate frame of the assembly and based on the tolerance scheme as specified in tolerance specification.

\subsection{FROOM}

FROOM is a prototype of a re-design support system, currently under development. FROOM is an acronym for feature and relation based object oriented modeling. For more details on FROOM, refer to [1,2] as well as part I (the tolerance specification paper).

\subsection{Overview of the paper}

In Section 2 an overview is presented of previous work in tolerance analysis. The overall design of the tolerance analysis module of FROOM is presented in Section 3. In Section 4, the implementation is elaborated. The results achieved with this implementation are discussed in Section 5 and finally in Section 6, conclusions and recommendations are given.

\section{Previous work}

In this section previous work in the field of tolerance analysis is described. One of the first who proposed a method for tolerance analysis was Bjørke [3]. Bjørke states that some dimensions are more important than other dimensions. These dimensions. called sum-dimensions, are dependent on the individual dimensions. The relation between both dimension types is deduced from a tolerance chain. Based on the variation of the individual dimensions, the resulting value for the sum-dimension is calculated. The method which is only applicable for $2 \mathrm{~V}$ mechanisms, is highly depending on manufacturing process parameters and only takes size tolerances into account.

In $[4,5]$, a method is proposed to calculate the contact states of two parts with respect to each other. First, the parts are assumed to have their nominal shapes and are located at their ideal positions. Then deviation models are produced by slightly changing the positions of the nominal shapes. An algorithm now calculates the contact states in the assembly. In this method, only feasibility of assembly is accounted for and tolerances are not treated as zones.

Clément et al. [6-9] present a general model in which surface associations play an important role, the so-called Technologically and Topologically Related Surfaces (TTRS) approach. In this approach, tolerances are treated as small displacements which are in turn described with a tolerance torsor. Thus the tolerances are described in a highly mathematical way. This method, with some adaptations, has been implemented in FROOM; see the tolerance specification paper (part I) and $[1,2,10]$. Based on the TTRS approach two methods 


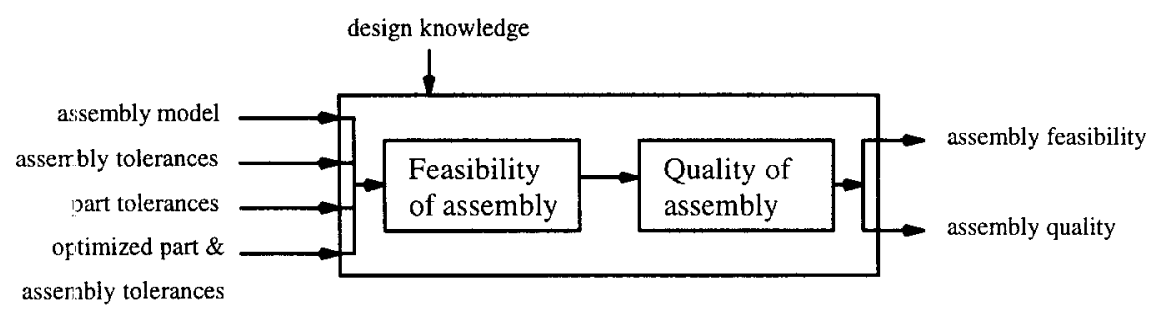

Fig. 1. Main function of the tolerance analysis tool.

have been developed for tolerance analysis. Gaunet uses tolerance torsors to represent the tolerance zones [11]. Rivière et al. use matrices similar to the homogeneous coordinate transform matrices that are well known in robotics to determine the resulting tolerance zones [12]. In this paper the methods are referred to as torsor approach and matrix approach respectively. Both the torsor approach and the matrix approach allow the calculation of clearances and orientations in an assembly.

\section{Overall functionality of the tolerance analysis tool}

A first design of the functional tolerancing module has been described in $[1,2,10]$. As stated before, tolerance specification has already been implemented in FROOM. Tolerance analysis and tolerance synthesis can be seen as complementary constraint satisfaction methods after the tolerances have been specified. The main function of tolerance analysis can be defined as:

Calculate with given tolerance types and tolerance values, the resulting tolerance zones in order to verify the mating function of the parts constituting the assembly (feasibility of assembly) and calculate the clearances and orientations between the parts constituting the assembly (quality of assembly).

In order to fulfill the main functions of tolerance analysis, shown in Fig. 1, several working principles have to be selected. Due to space limitations this is not addressed in this paper. Interested readers are referred to [13]. The calculation of softgages can be used in feasibility of assembly as described by Gaunet [11]. The matrix approach by Rivière et al. [12] has been selected for implementation of quality of assembly in FROOM. Several reasons can be given for this. First, using matrices instead of torsors reduces the number of equations. The use of torsors requires the calculation of the displacement in a lot of points in the tolerance zone [11]. However, there will always be one equation that restricts the tolerance torsor most [10]. In the matrix approach only a limited number of points is needed [10]. Second, the use of matrices is a more common approach than the use of torsors, at least in Anglo-Saxon literature. The matrix approach is extended with a method to reduce the number of parameters in the set of equations, based on an analogy to the plan fragments used in degrees of freedom (DOF) analysis [14]. Simulated annealing (SA) is used as a method to solve the resulting reduced set of equations.

In Section 3.1, the matrix approach, including a method for reducing the number of equations, is treated in more detail. A method to reduce the number of parameters is provided in Section 3.2. In Section 3.3, a method is proposed to solve the derived set of equations, followed by an example in Section 3.4.

\subsection{Matrix approach}

In the matrix approach tolerance zones can be represented mathematically by a matrix, called matrix $D$, which describes the small displacements in the tolerance zones [12]. The final displacements in the tolerance zones, have to be calculated with respect to a datum surface. A datum surface should be selected for each 


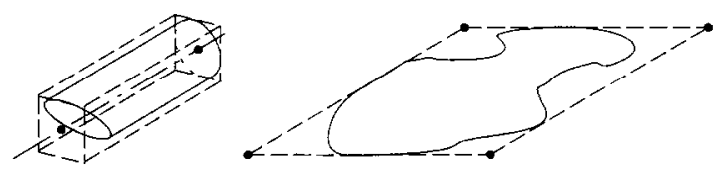

Fig. 2. Points needed in an irregularly shaped cylinder (a) and planar plane (b).

clearance or orientation calculation. This datum surface must be part of the loop in which the assembly condition appears. This means that all tolerance zones are calculated with respect to this reference. In general, the tolerance zones are defined with respect to a local coordinate frame. The matrix $P$ represents the transformation of the local coordinate frame to the coordinate frame of the datum surface, $R$. The vector of displacements, $M M^{\prime}$, of a point $M$ in Euclidian space to $M^{\prime}$, representing the displacement in the tolerance zone and expressed in the local coordinate frame $R_{1}$ is [12]:

$$
\left[M \vec{M}^{\prime}\right]_{R_{1}}=(D-I) *\left[P_{R \rightarrow R_{1}}\right] *[M]_{R}
$$

In order to define formula (1) for each tolerance zone, a number of points is needed, to describe the displacements. The number of resulting equations is equivalent to the number of points. The number and the location of these points largely depends on the TTRS case [13]. These points should be the most extreme points of the surface itself according to some criterion. For instance the vertices of the bounding box of the surface in case of irregular shapes, or the intersection of MGDE and the bounding box associated with the surface. Using these points, the number of equations is reduced compared to [11], as has been shown in [10,13]. In Fig. 2, some examples are given. For an irrcgularly shaped cylinder (Fig. 2(a)) the two intersection points of the axis of the cylinder (MGDE) and the bounding box are chosen to describe the displacement in the tolerance zone, so only two equations are generated. For an irregularly shaped planar plane (Fig. 2(b)) a bounding box is made and on the corners of this bounding box, the displacements are calculated. Although this may in some cases result in an exaggerated worst case analysis, these assumptions greatly reduce the number of equations to be dealt with. Using these points, the tolerance constraints are defined as [12]:

$$
\left[M_{\text {surface, point }} \vec{M}_{\text {surfacc, point }}^{\prime}\right]_{R_{1}}^{T} \cdot\left[\begin{array}{c}
x_{1} \\
y_{1} \\
z_{1} \\
0
\end{array}\right] \leq \frac{\text { tolerance value }}{2} .
$$

The next step is to define the maximization or minimization function for the assembly condition. A distinction should be made between the calculation of maximum and minimum clearances and the several cases of orientation which are possible for the surfaces considered. For clearances, only optimization functions are necessary. For orientation calculations, additional constraints are needed to describe the orientation of the surfaces or MGDEs in the several orientation cases. Consider for example the clearance and orientation between two cylinders in parallel. For each of the cases, a maximization or minimization function can be defined, including additional constraints for orientation, slightly adapted after [12]:

$\begin{array}{ll}\text { Maximum clearance: } & \text { maximize }\left|e_{1 a}-e_{2 a}\right| \\ \text { Minimum clearance: } & \text { maximize }\left|-e_{1 a}+e_{2 a}\right|\end{array}$ 


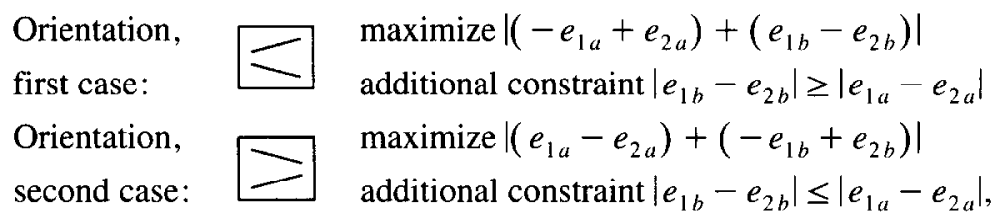

where $e_{i}$ is the displacement in the points of the surfaces involved in the clearance or orientation which has to be calculated. A set of constraints and additional constraints could be defined for each tolerance case.

\subsection{Virtual plan fragment direction}

As stated before, a main disadvantage of the method by [11] is that sets of equations, containing a lot of parameters, are generated in clearance and orientation calculations. Although, the matrix approach is able to reduce the number of equations, it seems worthwhile to investigate a method in which the number of parameters of these sets is also reduced. Another reason is that it is difficult to find the directions in each tolerance zone of a pseudo TTRS which influence the most the clearance one is interested in. A combination of the matrix approach and reasoning about macro-DOFs has been investigated, in order to reduce the number of parameters in the set of equations. The macro-DOFs are the rotations and translations an object can have without affecting the nominal geometric constraints imposed on the object. A reduction of the number of equations is also possible, when a closed path of force is part of the tolerance chain. In closed paths of force the clearance is zero because a force is applied on the surfaces to maintain contact.

The method to reduce the number of parameters in the set of equations is based on virtual plan fragments. The term plan fragment was originally introduced by Kramer [14]. Kramer uses plan fragments to solve nominal geometric constraints. In this method, the degrees of freedom, the constraint type and the geometric configuration are used in satisfying the constraints. The translations and rotations of an object, necessary to fulfill a constraint between two objects, are described by the plan fragments. These displacements are referred to as macro-DOFs to distinguish them from small displacements, or micro-DOFs, which are allowed by the tolerance zones. The macro-DOFs can be used to determine the most critical direction for an assembly condition, a micro-DOF.

For tolerance analysis, Kramer's theory can be used in a somewhat different way. First, the loops in which the assembly conditions appear are detected. The objects for which the assembly condition is specified are called the primary cibjects. Now, for each assembly condition the virtual plan fragment direction is calculated. This direction represents how the primary objects should be displaced within their respective tolerance zones to obtain the maximurn or minimum clearance or orientation. Parameters describing displacements in directions other than the most critical direction can be removed from the tolerance constraint and so the number of parameters in the set of equations can be reduced.

For each loop, the clearance or orientation is now calculated. Therefore, the loop is divided into two chains. In a chain, the traversal from one of the primary objects to the datum surface on the base object, the object having zero macro-DOFs, is represented. First, only the virtual plan fragment direction of the primary objects is considered in the tolerance chain. After each traversal to a next component in the tolerance chain a check is performed in order to determine if it is still possible to consider the virtual plan fragment direction of the primary objects. When all loops in which the assembly condition appears are traversed, the most restrictive loop can be determined. 'The above is illustrated in Fig. 3.

A table is necessary to determine the virtual plan fragment direction for each combination of elementary surfaces. The virtual plan fragment table is based on the reclassification of Clement's table [6,7], because the orientation of the surfaces will be of importance in determining the virtual plan fragment directions. Two virtual plan fragment tables have been developed, one for clearances and one for orientations [13]. 


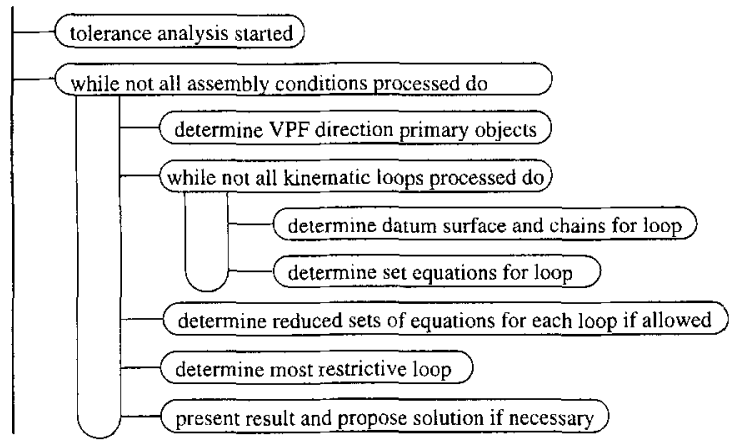

Fig. 3. Schematic algorithm for analyzing the quality of assembly.

The virtual plan fragment directions can in general be determined best by first determining the pseudo TTRS case. In the virtual plan fragment table these cases are listed and using the contents of the table the virtual plan fragment direction can be determined. This can be illustrated by the example of Fig. 4.

In Fig. 4(a), the virtual plan fragment direction for two parallel cylinders is determined. The virtual plan fragment direction is determined by calculating the normal vector of both axes of the cylinders. In Fig. 4(b), the virtual plan fragment direction for two concentric cylinders is determined. As can be seen from the figure, the virtual plan fragment direction is determined by calculating the normal vectors of the axes of the cylinders. In this case, there is an infinitesimal number of directions, because the cylindric tolerance zone is symmetric. So, an additional criterion is necessary. When a virtual plan fragment direction is already available and if it coincides with one of the possible directions in the 2D plane (Fig. 4(b)), this direction can be chosen. On the other hand, when no virtual plan fragment direction is available, a direction which corresponds with a direction of the datum reference frame can be chosen, or a direction can be chosen depending on the next association in the chain. For each pseudo 'I"IRS case the virtual plan fragment directions can be deduced. The virtual plan fragment table for clearances is presented in Fig. 5. For a more detailed description of the different cases of the virtual plan fragment tables readers are referred to [13].

The clearance or orientation is calculated using formulas (1) and (2). In these formulas, the number of parameters should be reduced depending on the virtual plan fragment directions for the assembly condition. The elements in formulas (1) and (2) that have to be changed are $[D-I]$ and $\left(x_{1}, y_{1}, z_{1}, 0\right)$. In $D-I$ the matrix $D$ describing the displacements in the tolerance zone has to be adapted. The translation and rotation parameters which do not lead to a displacement along the virtual plan fragment direction can be removed from the matrix.
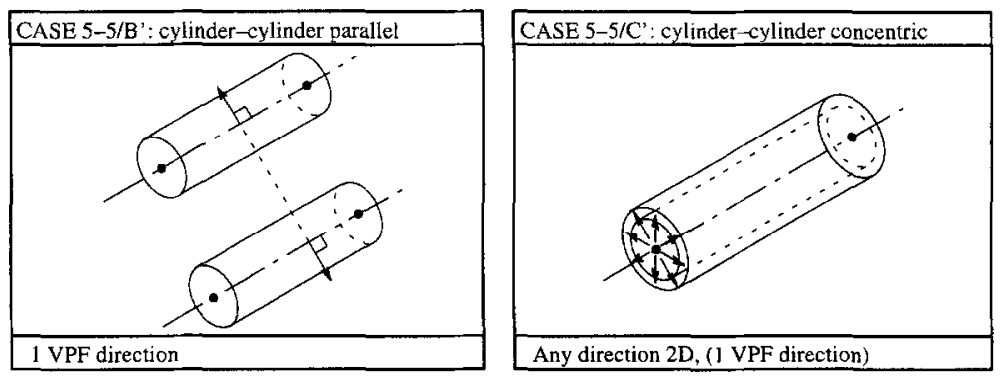

Fig. 4. Virtual plan fragment directions for two cylinders (in case of clearance). 


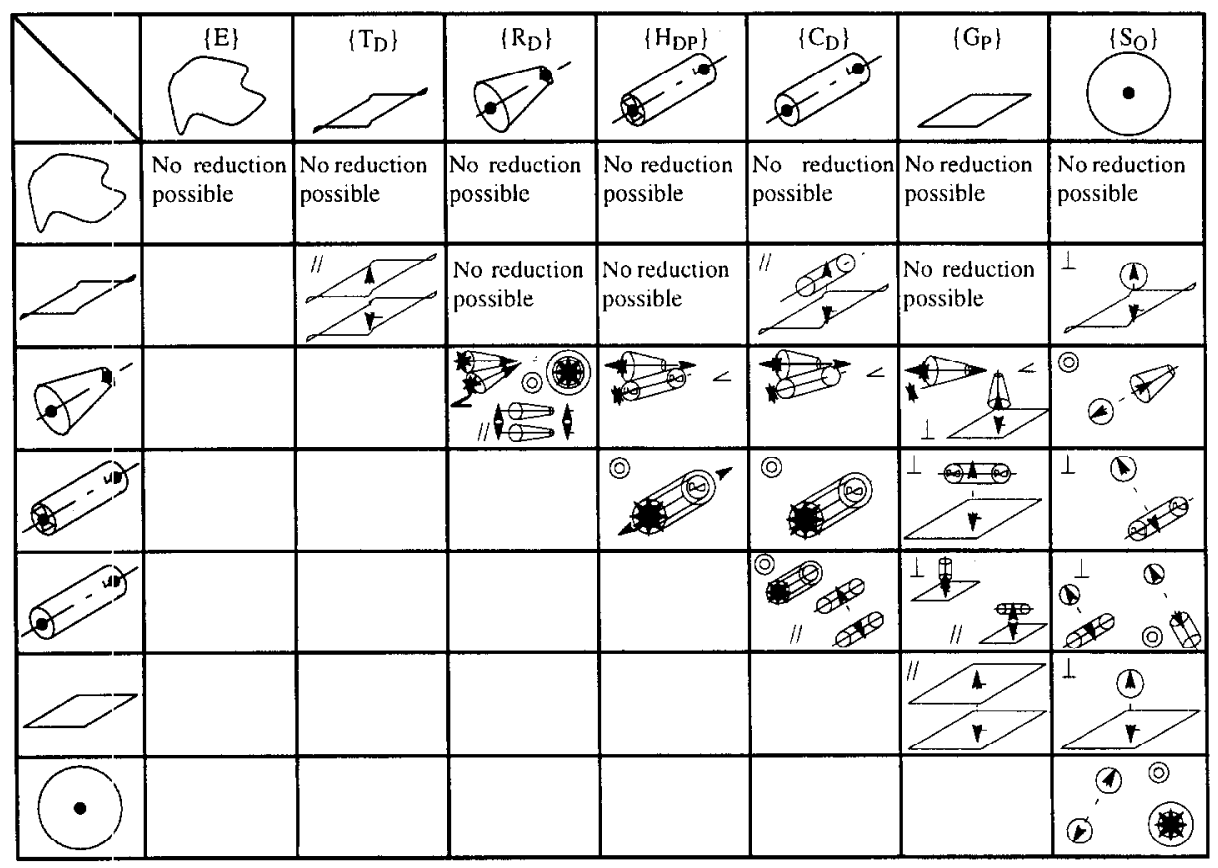

Fig. 5. Virtual plan fragment table for clearances.

For example, consider a cylindrical tolerance zone. The tolerance matrix (4) is then defined as shown in Fig. 6.

$$
D(\nu, w, \beta, \gamma)=\left[\begin{array}{cccc}
C \gamma C \beta & -S \gamma & C \gamma S \beta & 0 \\
S \gamma C \beta & C \gamma & S \gamma S \beta & \iota \\
-S \beta & 0 & C \beta & w \\
0 & 0 & 0 & 1
\end{array}\right]
$$

When the $y$-axis is defined as the virtual plan fragment direction for this example, the translation parameters working in the $z$-direction, $w$, can be removed from matrix (4). Apart from the translation parameters, also the number of rotation parameters can be reduced. Only the rotation parameters which lead to a displacement in the virtual plan fragment direction have to be considered. Therefore, $\beta$, the rotation around the $y$-axis, can be removed from matrix (4). The resulting matrix after reduction is then as follows [13]:

$$
D(v, w, \beta, \gamma)=\left[\begin{array}{cccc}
C \gamma & -S \gamma & C \gamma S & 0 \\
S \gamma & C \gamma & S \gamma & v \\
0 & 0 & 0 & 0 \\
0 & 0 & 0 & 1
\end{array}\right]
$$

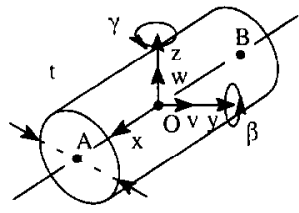

Fig. 6. Cylindric tolerance zone and associated displacements matrix (adapted after [12]). 
Vector $\left(x_{1}, y_{1}, z_{1}, 0\right)$ can also be reduced. This vector represents the direction of the displacement in the tolerance zone. When again the cylindrical tolerance zone is considered, the vector can be reduced to $(0,1,0$, 0 ). This implies that only the $y$-direction of formula (2) becomes part of the set of equations. For each TTRS and pseudo TTRS of the loop an equation is formulated and the final set of equations for the clearance or orientation is composed. This set does now have to be solved. In the next section a method is presented to solve and optimize the set of equations.

\subsection{Solving and optimizing the set of equations}

The set of equations should now be solved. Rivière et al. [12] do not propose any solutions in order to solve the set of equations. Traditional optimization methods, like hill descent algorithms, could be used to solve the set of equations. However, adaptive search methods like simulated annealing (SA) and genetic algorithms (GAs) have several advantages compared to these traditional optimization methods. One of the main advantages is that adaptive search algorithms have the ability to find the global optimal solution of an optimization problem because they have a random aspect. Three factors contributed to the selection of SA instead of GAs. First, the time taken to find a solution with SA was shown to be shorter compared to GAs, when applied to constraint satisfaction problems [15]. Second, SA seems especially attractive for solving non-linear, highly coupled inequality constraints [15]. Third, in FROOM, SA has already been implemented for solving parameter constraint satisfaction problems.

SA as described in [15] is suitable for the domain of constraint satisfaction problems. In tolerance analysis, a function has to be optimized with respect to a set of constraints. Therefore, SA had to be adapted to this specific domain for implementation in FROOM $[13,16]$.

\subsection{Example of a gearpump}

A gearpump has been selected as an example because it has also been used in other publications, e.g. [11,12]. In Fig. 7, the toleranced assembly and the associated assembly graph are shown. As can be seen from Fig. 7, only the loop $(D 1, A 2, B 2, B 3, A 3, B 1)$, in which the clearance appears is considered. From the virtual plan fragment table it can be derived that the $y$-direction is the virtual plan fragment direction for the primary objects. Surface $D 1$ is selected as datum surface. Now the loop is split into two chains: $(B 2, A 2, D 1)$ and $(B 3$, $A 3, B 1, D 1$ ). First, the tolerances on the primary objects, $B 2$ and $B 3$, have to be considered. In both tolerances, two concentric cylinders are involved. The virtual plan fragment direction is then undetermined at first, see Fig. 4. Because the $y$-direction has already been determined as virtual plan fragment direction for the primary objects, the virtual plan fragment direction for the tolerances on the primary objects is also the $y$-direction. Matrices $D_{22}$ and $D_{33}$ can therefore be reduced, when the chains are completely traversed and reduction turns out to be allowed. The next step is the calculation of the tolerance constraints according to formulas (1) and (2). For $D_{33}$ the reduced tolerance constraint becomes $[12,13]$ :

$$
\left(\left[P_{R \rightarrow R_{5}}\right]^{-1} *\left[D_{33}-I\right] *\left[P_{R \rightarrow R_{5}}\right] *\left[M_{B 3}\right]_{R}\right) \cdot \vec{y} \leq \frac{\text { tol5 }}{2} \Rightarrow c_{1} \cdot v+c_{2} \cdot \gamma \leq \frac{\text { tol5 }}{2},
$$

where $R_{i}$ (with in this case $i=5$ ) again represents the local and $R$ the global coordinate frame. The traversal to the next component can now be performed in both chains. An evaluation takes place whether the virtual plan fragment direction is still valid. Because two parallel cylinders, having the same orientation as the primary objects are associated, the virtual plan fragment direction is still directed along the $y$-direction in both chains. 

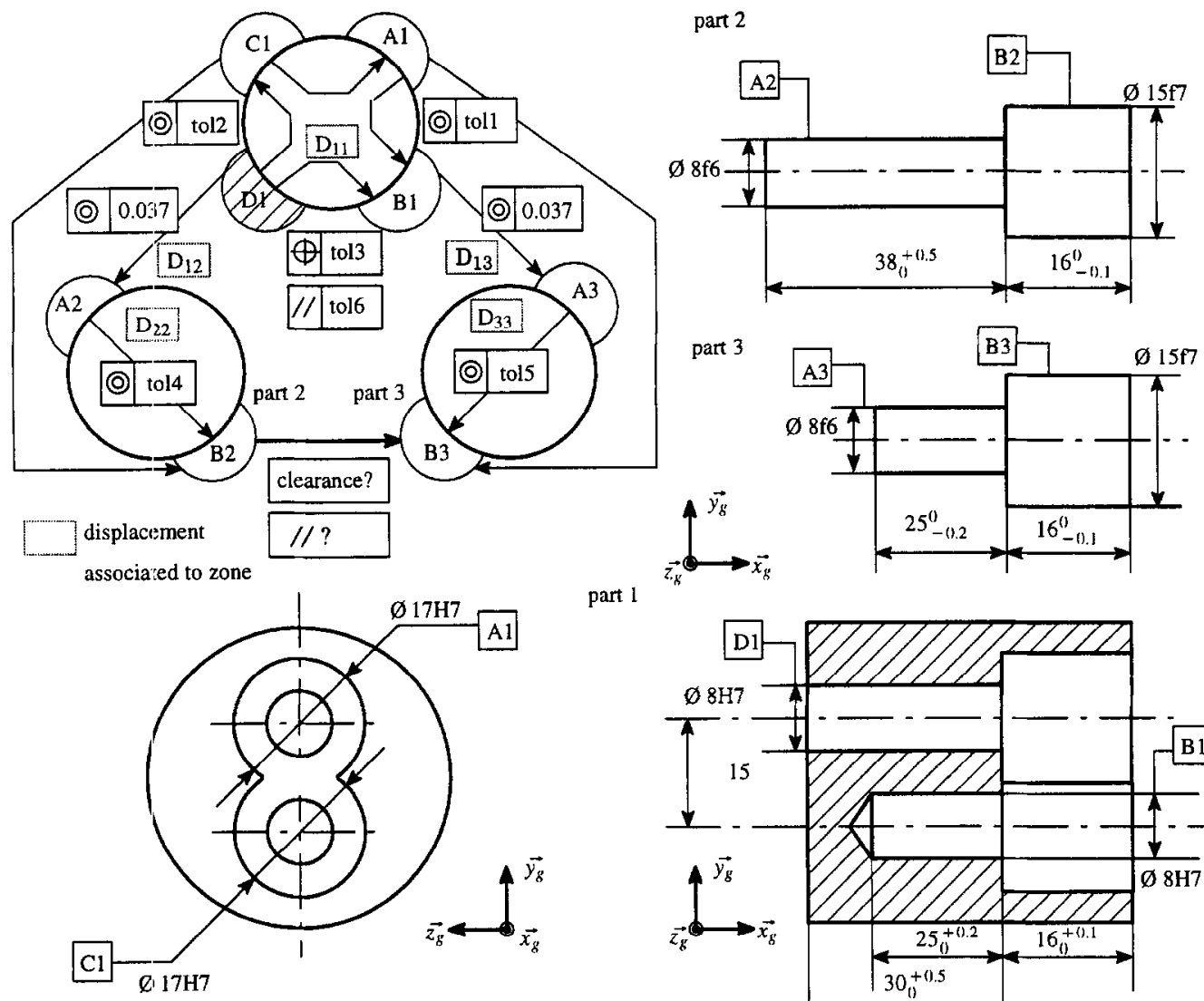

Fig. 7. The gearpump (adapted after [12]).

Thus, the reduction can be maintained and formulas can be formulated for the small displacements of the primary objects [12]:

$$
\begin{aligned}
e_{2}= & \left(\left[P_{R \rightarrow R_{2}}\right]^{-1} *\left[D_{12}-1\right] *\left[P_{R \rightarrow R_{2}}\right] *\left[M_{A 2}\right]_{R}\right) \cdot \vec{y} \\
& +\left(\left[P_{R \rightarrow R_{4}}\right]^{-1} *\left[D_{22}-I\right] *\left[P_{R \rightarrow R_{4}}\right] *\left[M_{B 2}\right]_{R}\right) \cdot \vec{y}, \\
e_{3}= & \left(\left[P_{R \rightarrow R_{1}}\right]^{-1} *\left[D_{11}-I\right] *\left[P_{R \rightarrow R_{1}}\right] *\left[M_{B 1}\right]_{R}\right) \cdot \vec{y} \\
& +\left(\left[P_{R \rightarrow R_{3}}\right]^{-1} *\left[D_{13}-I\right] *\left[P_{R \rightarrow R_{3}}\right] *\left[M_{A 3}\right]_{R}\right) \cdot \vec{y} \\
& +\left(\left[P_{R \rightarrow R_{5}}\right]^{-1} *\left[D_{33}-I\right] *\left[P_{R \rightarrow R_{5}}\right] *\left[M_{B 3}\right]_{R}\right) \cdot \vec{y}
\end{aligned}
$$

This set of equations can now be optimized using SA and formulas (3a) and (3b).

\section{Tolerance analysis in FROOM}

In this section, the implementation of the tolerance analysis module in FROOM is described. Based upon the theory as described above, FROOM is currently able to calculate the clearances for assemblies which are built 


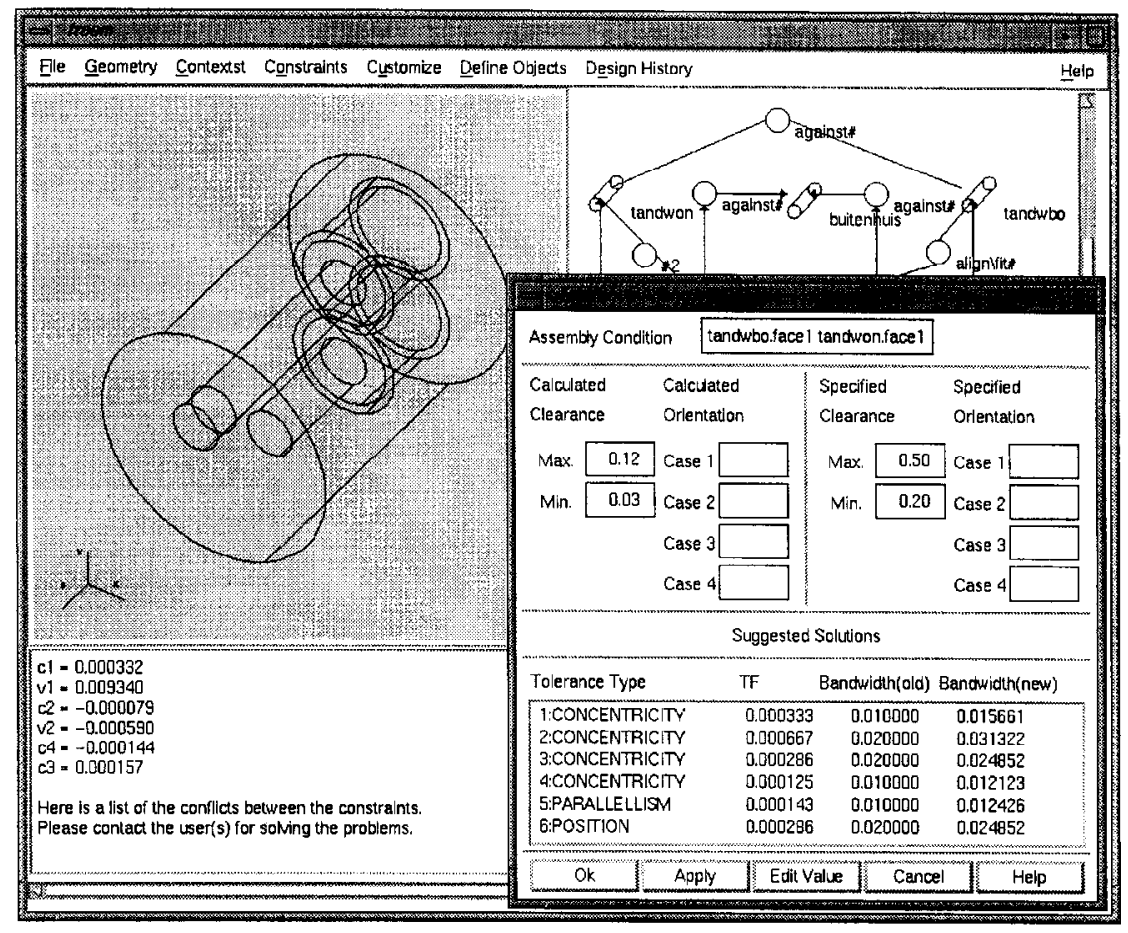

Fig. 8. The results and a solution are proposed to the designer.

of cylindrical faces, planar faces and conical faces. However, the module can easily be extended to other surface types. The module is implemented using $\mathrm{C}++$. An object oriented approach is used. The user interface is based on OSF $/$ motif $^{\mathrm{TM}}$ for X-Windows.

Fig. 8 provides an example of the results of a clearance calculation. As can be seen from the figure, apart from the results, a solution is presented to the designer if the clearance specifications are not met.

\section{Discussion of the results}

In the previous sections, a method for worst case quality of assembly has been presented. Although this method has successfully been implemented in FROOM, several aspects of the theory as presented in Section 3 have to be verified, such as the validity of the applied reduction of the number of equations as well as parameters. Thus, a verification of the possibility of an exaggerated worst case tolerance analysis is needed. Apart from this, the method has to be verified in true 3D clearance calculations and more complex assemblies. Although the gearpump which has been treated in Section 3.4 is a relatively simple example, it demonstrates the derived theory at least in $21 / 2 \mathrm{D}$ assemblies. The maximum and minimum clearances can be calculated. Due to its simplicity, the results obtained from the gearpump example can be interpreted and be compared to "analytical" results. The latter is possible because the clearances can also be calculated by substituting the tolerance values in the equation for the clearance. Because of the relatively simple geometry, the equation for the clearance can still be derived by hand in the case of the gear pump.

Finally, the results obtained with SA have to be verified. As stated in Section 3.3, the SA algorithm has been adapted to the combined domain of constraint solving and constraint optimization problems. Although the results seemed promising, they should be compared with a numeric iterative optimization method. 


\section{Conclusions and recommendations}

A tolerance analysis tool has been presented which allows for worst case tolerance analysis of assemblies as far as quality of assembly is concerned. An extension has been presented to the methods by Gaunet and Rivière et al., which restricts the number of equations as well as the number of parameters in the set of equations. The number of equations is restricted by considering a minimum number of points, depending on the TTRS case. The method to reduce the number of parameters reasons with the macro-DOFs and geometry in order to find the virtual plan fragment direction for the primary and secondary objects. Based on this direction, the number of parameters can be reduced. A third extension is the use of SA for solving and optimizing the set of constraints. However, these extensions should be verified in a more complex product model. The use of statistical methods should be investigated in addition to worst case tolerance analysis. The integration of the presented methods and statistical approaches could lead to a powerful tolerance analysis tool. Also, tolerance synthesis should be developed, in combination with the presented methods.

\section{Acknowledgements}

This research has been supported by the Technology Foundation (or STW: a Dutch foundation).

\section{References}

[1] O.W. Salomons, "Computer support in the design of mechanical products, constraint specification and satisfaction in feature based design for manufacturing”, Ph.D. Thesis, University of Twente, Enschede, The Netherlands, 1995.

[2] O.W. Salomons, H.J. Jonge Poerink, F. van Slooten, F.J.A.M. van Houten and H.J.J. Kals, “A tolerancing tool based on kinematic analogies", Computer Aided Tolerancing. Tokyo, Japan (Chapman and Hall, 1995) (F. Kimura, ed.).

[3] $\varnothing$. Bjørke, Computer Aided Tolerancing, Tapir Publishers, 1978.

[4] K. Takahashi, H. Suzuki and F. Kimura, "Tolerance analysis in machine assembly by classifying part contact state", CIRP International Working Seminar on Computer Aided Tolerancing, Pennsylvania State University, U.S.A., 1991.

[5] K. Takahashi, H. Suzuki and F. Kimura, "Motion analysis with geometrical errors based on dynamic simulation", CIRP International Working Seminar on Computer Aided Tolerancing, ENS de Cachan, Paris, France, April 1993, pp. 85-95.

[6] A. Clément, A. Dissrochers and A. Rivière, "Theory and praclice of 3D tolerancing for assembly", CIRP International Working Seminar on Comptter Aided Tolerancing, Pennsylvania State University, U.S.A., 1991.

[7] A. Clément and A. Rivière, "Tolerancing versus nominal modelling in next generation CAD/CAM systems", CIRP International Working Seminar on Computer Aided Tolerancing, ENS de Cachan, Paris, France, April 1993, pp. 97-113.

[8] A. Clément, A. Rivière and M. Temmerman, Cotation tridimensionelle des systèmes méchaniques, théorie et pratique, PYC Edition, Ivry-sur-Seine Cedex, 1994 (in French).

[9] A. Desrochers and A. Clément, "A dimensioning and tolerancing assistance model for CAD/CAM systems", International Journal of Advanced Manufacturing Technology, Vol. 9, 1994, pp. 352-361.

[10] H.J. Jonge Poerink., "A functional tolerancing module for FROOM”, M.Sc. Thesis, PT-516, University of Twente, Enschede, The Netherlands, 1994.

[11] D. Gaunet, "Modèle formel de tolérancement de position, contributions à l'aide au tolérancement des mécanismes en CFAO", Ph.D. Thesis, L'école Supérieure de Cachan, Paris, 1994 (in French).

[12] A. Rivière, D. Gaunet, I. Dubé and A. Desrochers, "Une approche matricielle pour la représentation des zones de tolérance et des jeux", Proceedings FORUM 1994 de la SCGM (Société Canadienne de Génie Mécanique), McGill University, $27-29$ June, 1994.

[13] F.J. Haalboom, '“Tolerance analysis in FROOM"', M.Sc. Thesis, PT-549, University of 'Twente, Enschede, The Netherlands, 1995.

[14] G.A. Kramer, Solving Geometric Constraints Systems: A Case Study in Kinematics, The MIT Press, Cambridge, Massachusetts, 1992.

[15] A.C. Thornton and A. Johnson, "Constraint specification and satisfaction in embodiment design", Proceedings International Conference on Engineering Design (ICED '93), The Hague, The Netherlands, 17-19 August, 1993, pp. 1319-1326.

[16] F.J. Haalboom, Tolerance Analysis in FROOM: Software Documentation, Internal Report PT-569, University of Twente, Enschede, The Netherlands, 1995. 


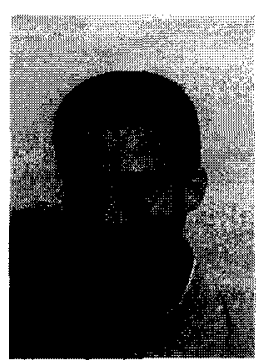

O.W. Salomons obtained a Ph.D. degree on his work on the FROOM system at the University of Twente in 1995. He obtained his M.S. degree in Mechanical Engineering in 1990 at the same University. Presently, as an Assistant Professor, he is performing research in the field of design support systems as well as their link with process planning systems.

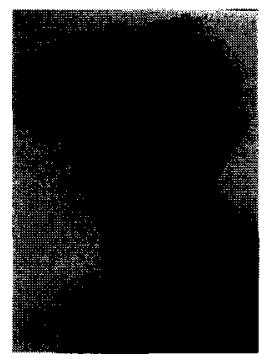

F.J. Haalboom obtained his M.S. degree in Mechanical Engineering at the University of Twente in 1995. His M.S. work was on the tolerance analysis module in the FROOM system. Currently he is employed at CAP-Volmac.

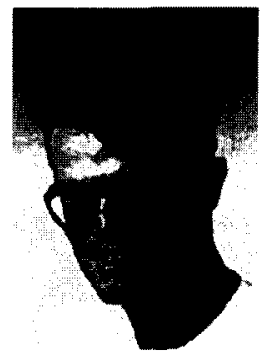

H.J. Jonge Poerink obtained his M.S. degree in Mechanical Engineering at the University of Twente in 1994. His M.S. work was on the tolerance specification module in the FROOM system. Currently he is employed at Philips Machinefabrieken.

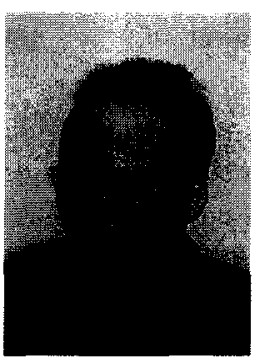

F. van Slooten holds a B.S. in Computer Science, which he obtained in 1990. Since 1991 he works as a System Analyst / Technical Software Developer at the Laboratory of Production and Design Engineering of the University of Twente.

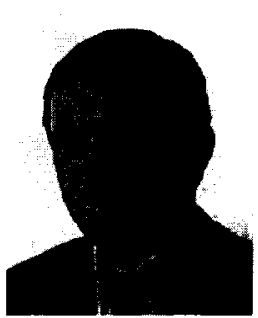

F.J.A.M. van Houten is Associate Professor of the Laboratory of Production and Design Engineering at the University of Twente. Professor Van Houten obtained his M.S. degree in Mechanical Engineering at the Technical University of Eindhoven in 1976. He has been working at the Laboratory of Production Engineering at University of Twente since 1978. Professor Van Houten has worked in the field of CAD and CAPP; he has been closely involved with the development of several CAPP systems. In 1991 he obtained a Ph.D. degree on his work on the PART system.

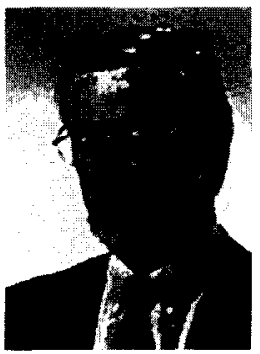

H.J.J. Kals is Professor and Head of the Laboratory of Production Engineering at the University of Twente. He is also part-time Professor at the Technical University of Delft. Professor Kals obtained his M.S. degree in Mechanical Engineering in 1969 at the Technical University of Eindhoven. In 1972 he obtained his Ph.D. degree. In 1977 he became professor of the Laboratory of Production and Design Engineering at the University of Twente. Professor Kals is a member of CIRP. He is active in the fields of CAD, CAPP, CAM, workshop- and work station control. Currently one of his main scientific interests is concurrent engineering. 